\title{
Palm Print Recognition using Zernike Moments
}

\author{
Subhajit Karar \\ Information Technology \\ Jadavpur University \\ Kolkata, West Bengal
}

\author{
Ranjan Parekh \\ School of Education Technology \\ Jadavpur University \\ Kolkata, West Bengal
}

\begin{abstract}
This paper proposes an automated system for recognizing palmprints for biometric identification of individuals. Complex Zernike moments are constructed using a set of complex polynomials which form a complete orthogonal basis set defined on the unit disc. Palmprint images are projected onto the basis set resulting in a set of complex signals. The magnitude of the complex value is computed and a scalar value is derived from it by computing the mean of the vector elements. Classification is done by subtracting the test samples from the mean of the training set. The data set consists of 80 images divided into 4 classes. Accuracy obtained is comparable to the best results reported in literature
\end{abstract}

\section{General Terms}

Pattern Recognition, Computer Vision

\section{Keywords}

Zernike moment, Palmprint recognition, Texture classification

\section{INTRODUCTION}

Biometrics refers to automatic recognition of individuals based on their physiological and behavioral characteristics, which are nowadays used extensively for personal identification worldwide. Different physical traits like face, iris, fingerprint, palmprint, retina etc. fall under the perview of biometrics. Palmprint recognition involves identifying an individual by the principal lines, wrinkles, ridges on the surface of the palm. The basis for using palmprints lies in the fact that no two individuals have exactly the same palmprint pattern, moreover palmprints remain more or less stable throughout the lifetime and are easily obtainable using standard imaging techniques. Challenges in palmprint recognition are related to building a reliable data model from randomly oriented irregular lines that enable high amount of accuracies in security based systems and applications.

This paper presents an efficient algorithm for palm print recognition by utilizing complex Zernike moments. The organization of the paper is as follows: section 2 provides an overview of the related works, section 3 outlines the proposed approach, section 4 details the experimentations done and results obtained, section 5 analyses the current work vis-à-vis contemporary works, section 6 brings up the overall conclusions and future scopes.

\section{RELATED WORKS}

Moments and functions of moments have been used as pattern features in a number of applications. Some of the earliest works include $[1,2]$. Zernike moments are a class of complex moments known for their invariance properties. In [3] the authors propose a palm print verification system using high order Zernike moments with k-nearest neighbour (k-NN) classifiers. Total 625 images are used from Hong Kong PolyU palm print database. In this paper the reported accuracy is
98\%. In [4] palm print recognition algorithm is based on Robust Oriented Hausdorff Similarity (ROHS) measure. Total 7752 palm images of Poly Technique university of Hong Kong is taken as dataset. In [5] the authors propose a contactless palm print principal line-based feature extraction technique. Here DFT technique is used to calculate distances from endpoints to endpoints and point of interception to endpoints. Correlation technique, power spectral matching and Euclidean distance are used as classifier. 100\% accuracy is reported in this paper. In [6] a hierarchical multi-feature scheme is used. Two levels of features are defined: geometry feature based on distance (level-1 feature) and texture feature based on Zernike moment (level-2 feature). Classification is done with two different neural networks and the output is combined into one recognition system. In [7] both geometrical and palm-print hand features are used. Texture extractions are done using wavelet transform, 2D Gabor filter and derivative methods. Support Vector Machine (SVM) classifiers are used as identifier and verifier. In [8] an efficient algorithm is used to extract the Region of Interest (ROI). The images are made geometric invariant. In [9] feature extractions are done using Gabor transform and Genetic Algorithm based on the specificuser. The palmprint images are used from Biological Research Center of Hong Kong Polytechnic University (PolyU). In [10] a palm print identification is done using wavelet transforms. The wavelets used for the analysis are Biorthogonal, Symlet and Discrete Meyer. Total 500 images are taken as dataset. In [11] the authors propose an innovative touch-less palm print recognition system. Here local binary pattern (LBP) method is used for feature extraction. Classification is done using a modified probabilistic neural network (PNN). In [12] the authors propose a principal line based palm print verification technique. Here a modified finite Radon transform is used for feature extraction. Pixel-to-area comparison method is used as classifier. In [13] the authors propose a palmprint recognition method using multiple correlation filters. Two ways of edge detection of images are done here. Firstly computing edginess of image of the palm print and secondly using phase symmetry processing of the image the edge is detected. 385 classes are used as the dataset. In [14] three orthogonal moments namely Zernike moments, pseudo Zernike moments and Legendre moments are used for feature extraction from palmprint images. Pseudo Zernike moments of order of 15 produce best result. Using this moment's accuracy is achieved $95.75 \%$. In [15] higher order Zernike moments is used for hand geometry feature extraction and the Log Gabor filter is used for palm print feature extraction. Overall accuracy achieved by combining these two features.

\section{PROPOSED APPROACH}

This paper proposes an efficient algorithm to recognize palm print images using Zernike moment with a scalar feature representation. 


\subsection{Zernike Moments}

Moments and functions of moments have been used as pattern features for several years. Regular moments have been the most popular types of moments. They are defined as follows where $m_{p q}$ is the $(p+q)$-th order moment of the image function $f(x, y)$

$$
m_{p q}=\sum_{x} \sum_{y} x^{p} y^{q} f(x, y)
$$

Unfortunately the basis $x^{p} y^{q}$ set of regular moments is not orthogonal. Consequently the recovery of image information from these moments is computationally expensive. Teague [16] has suggested the idea of orthogonal moments based on the theory of orthogonal polynomials to overcome this problem. Zernike moments are a class of such orthogonal moments. One advantage of Zernike moments is their rotational invariance i.e. rotating an image does not change the magnitude of its Zernike moments. Another advantage is the relative ease with which the image can be reconstructed from the moments. Zernike moments can also be constructed to an arbitrary high order.

In [17] Zernike introduced a set of complex polynomials which form a complete orthogonal set over the interior of the unit circle $x^{2}+y^{2}=1$. The form of these polynomials is given by

$$
V_{n m}(x, y)=V_{n m}(\rho, \theta)=R_{n m}(\rho) \exp (j m \theta)
$$

Here

$n \quad$ Positive integer or zero

$m \quad$ Positive and negative integers subject to the constraint $n-|m|$ even, $|m| \leq n$

$\rho \quad$ Length of vector from origin to $(x, y)$ pixel

$\theta \quad$ Angle between vector $\rho \& x$-axis in CCW direction

$R_{n m}(\rho)$ Radial polynomial defined as below, where

$k=n-|m| / 2$
$R_{n m}(\rho)=\sum_{s=0}^{k}(-1)^{s} \frac{(n-s) !}{s !\left(\frac{n+|m|}{2}-s\right) !\left(\frac{n-|m|}{2}-s\right) !} \rho^{n-2 s}$

Zernike moments are the projection of the image function onto these orthogonal basis functions. The Zernike moment of order $n$ with repetition $m$ for a continuous image function $f(x, y)$ that vanishes outside the unit circle is

$$
Z_{n m}=\frac{n+1}{\pi} \sum_{x} \sum_{y} f(x, y) V_{n m}^{*}(\rho, \theta), \quad x^{2}+y^{2} \leq 1
$$

To compute Zernike moments of an image, the center of the image is taken as the origin and pixel coordinates are mapped to the range of the unit circle. Pixels outside the unit circle are not used in the computations.

\subsection{Feature Values}

The real and imaginary parts of the 1-D complex Zernike moment computed from palmprint images, are separated out

$$
P=\operatorname{Re}(Z), Q=\operatorname{Im}(Z)
$$

The magnitude is calculated as follows

$$
S=P^{2}+Q^{2}
$$

The number of elements $N$ in vector $S$ depends on the order of the Zernike moments. A scalar $U$ is derived from the vector $S$ by computing the mean of its elements

$$
U=\frac{1}{N} \sum_{i=1}^{N} S_{i}
$$

The scalar value $U$ is subsequently used as the feature value corresponding to each image in the dataset. Classification is done by dividing the dataset into a training set $T$ and a testing set $S$ each consisting of $n$ samples. The $i$-th training class $T_{i}$ is represented by the mean of the feature values of all its component samples.

$$
M_{i}=\frac{1}{n}\left\{T_{i 1}^{F}+T_{i 2}^{F}+\cdots+T_{i n}^{F}\right\}
$$

The $j$-th test sample $S_{j}$ with feature value $S_{j}^{F}$ is classified to class $k$ if the absolute difference $D_{j, i}$ between the $j$-th test sample and $i$-th training class is minimum for $i=k$ i.e.

$$
S_{j} \rightarrow k, \quad \text { if } D_{j, i}=\left|S_{j}^{F}-M_{i}\right| \text { is minimum for } i=k
$$

\section{EXPERIMENTATIONS \& RESULTS}

Experiments are conducted by using 80 palm images from the PolyU palm print database [18]. The dataset is divided into 4 classes: A, B, C, D, each class consisting of 10 training images and 10 testing images. Each image is resized into 100 by 100 pixels in dimensions and in BMP format. Samples of training and testing images are shown in Figures 1 and 2
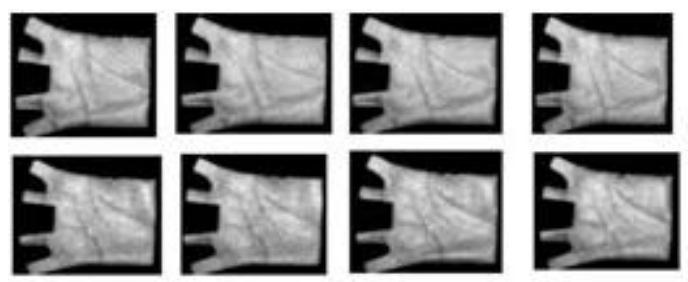

Class A
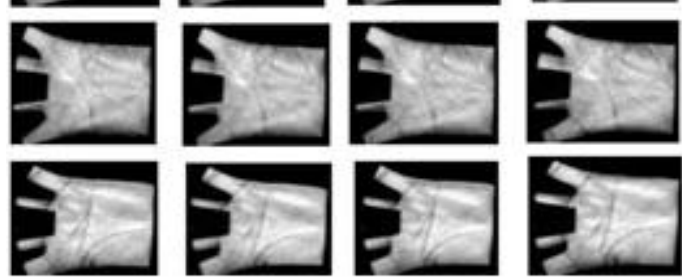

Class 8
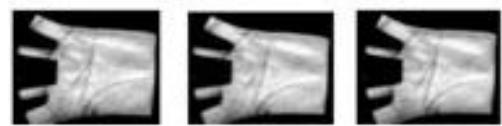

Class $\mathrm{C}$

Fig 1: Samples of Training Set Images
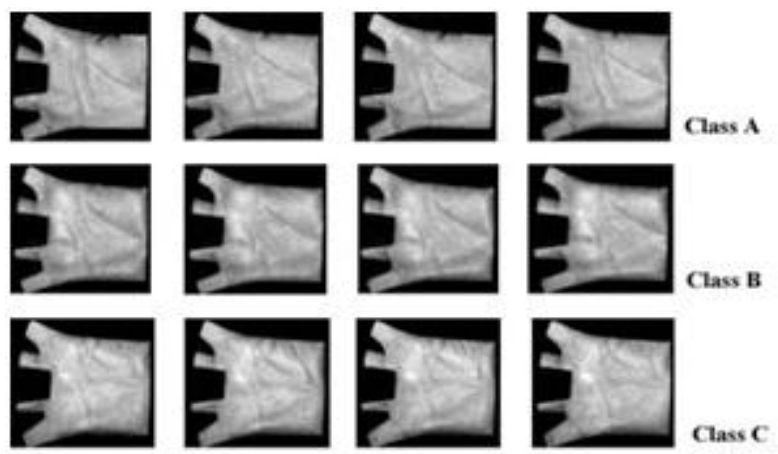

Class
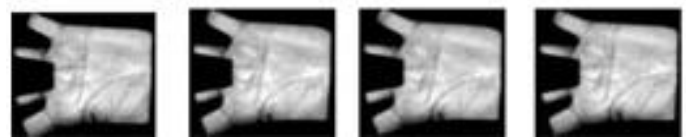

Class C

Fig 2: Samples of Testing Set Images 
Computations are done by varying order of Zernike moments from 1 to 10 . The best figures are obtained for orders 1,5 and 10. The corresponding feature plots for the training and testing sets, as well as the classification plots of these 3 cases are shown in figures $3,4,5$. For each figure, the upper subplot shows the variation of feature value $U$ for the training set files, the middle subplot shows the same for the testing set files, and the bottom subplot indicates the difference of the testing set files with the training set of each class. As expected for most cases, testing set files 1-10 belonging to Class-A shows the minimum difference with Class-A training set (blue), files 11-20 belonging to Class-B shows the minimum difference with Class-B training set (green), files 21-30 belonging to Class-C shows the minimum difference with Class-C training set (red), files $31-40$ belonging to Class-D shows the minimum difference with Class-D training set (cyan). The classification plots show the best accuracy value of $100 \%$ for order $1,95 \%$ for order 5 , and $90 \%$ for order 10 . Accuracies for other order values are indicated in Table 1.
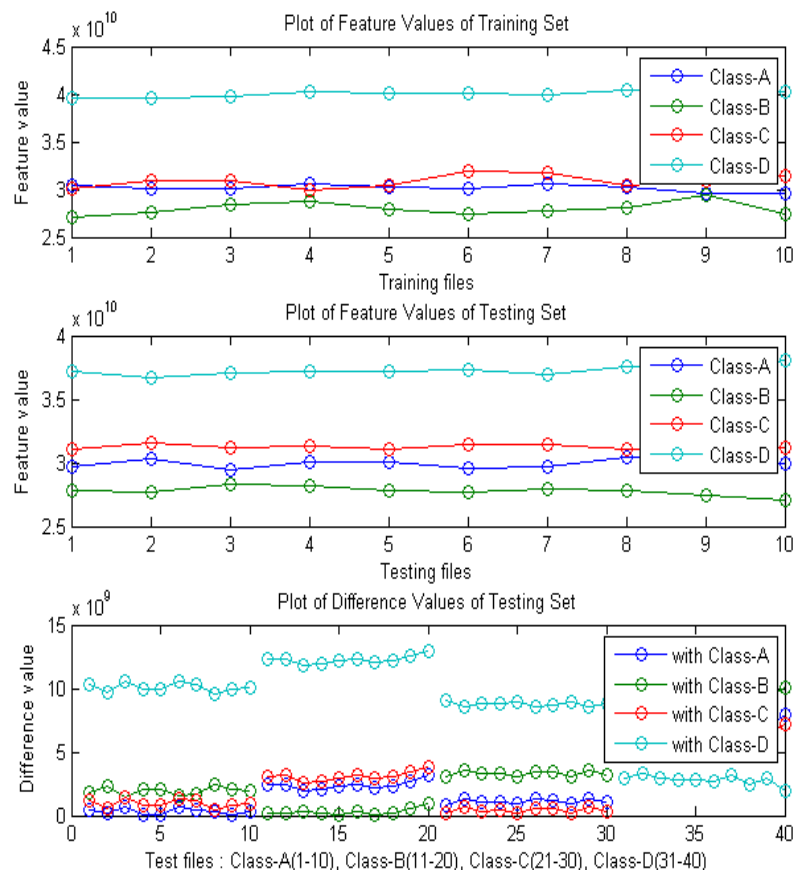

Fig 3: Feature and classification plots for order 1
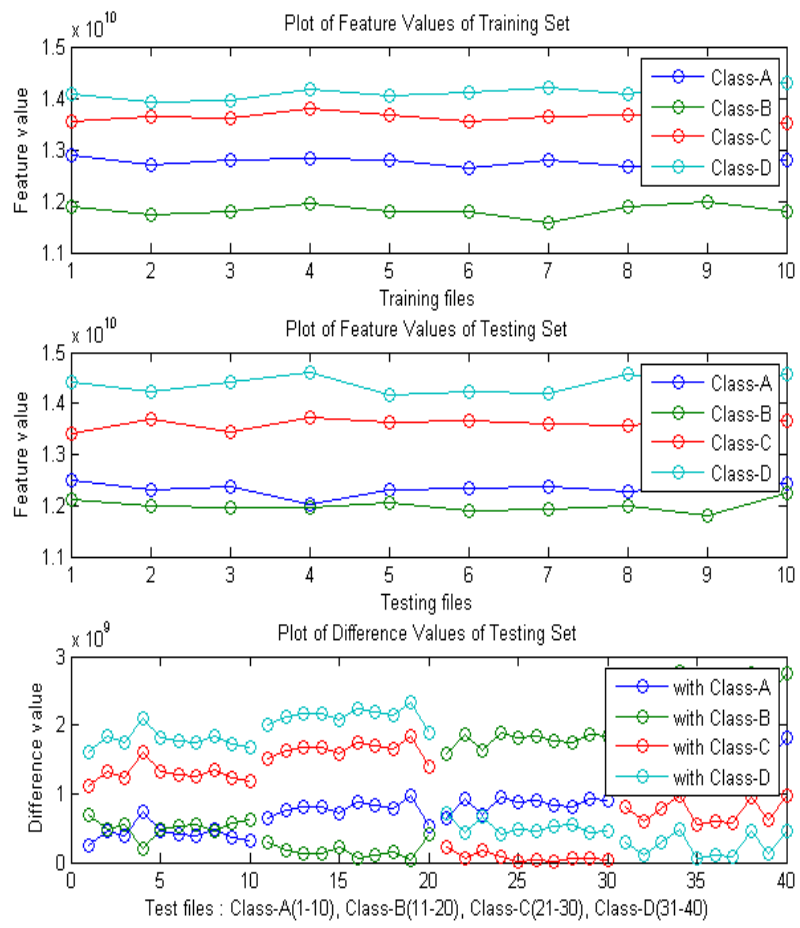

Fig 4: Feature and classification plots for order 5
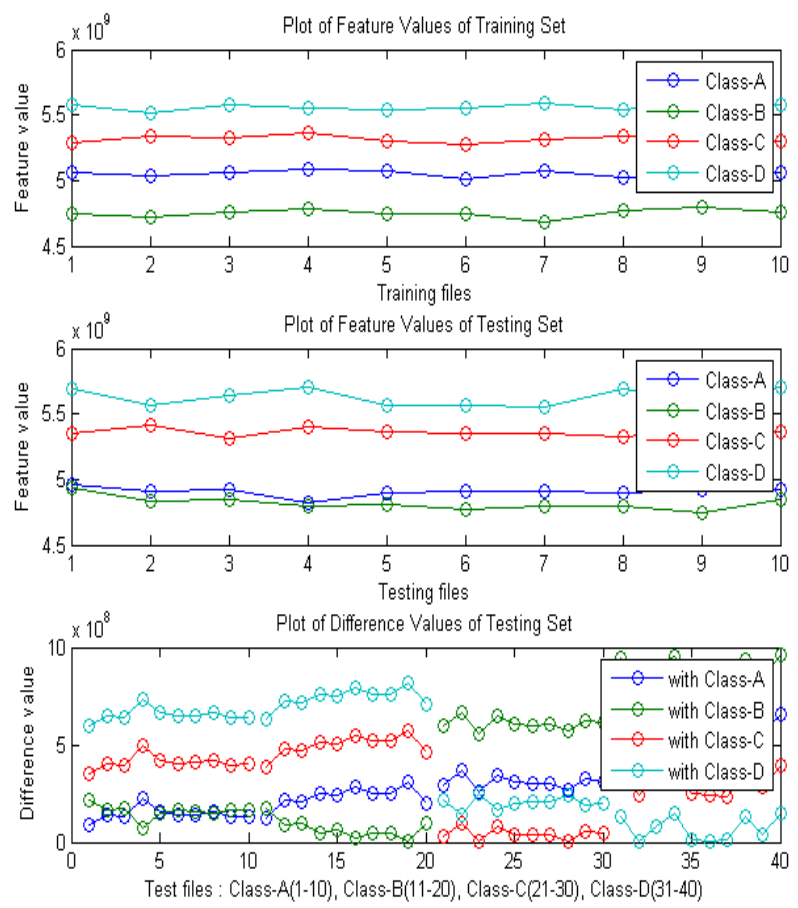

Fig 5: Feature and classification plots for order 10 
The accuracy values for the four classes are tabulated below in Table 1. The first column indicates the Zernike moment order, the second column shows the number of elements in the vector, the next four columns indicates the individual accuracy values for the 4 classes and the last column indicates the overall accuracy for all classes.

Table 1. Percentage Recognition Accuracies

\begin{tabular}{|c|c|c|c|c|c|c|}
\hline Order & $\mathbf{N}$ & $\begin{array}{c}\text { Class } \\
\mathbf{A}\end{array}$ & $\begin{array}{c}\text { Class } \\
\mathbf{B}\end{array}$ & $\begin{array}{c}\text { Class } \\
\mathbf{C}\end{array}$ & $\begin{array}{c}\text { Class } \\
\mathbf{D}\end{array}$ & $\begin{array}{c}\text { Overall } \\
\text { Accuracy }\end{array}$ \\
\hline 1 & 2 & 100 & 100 & 100 & 100 & 100 \\
\hline 2 & 4 & 90 & 80 & 100 & 0 & 67.5 \\
\hline 3 & 6 & 90 & 60 & 100 & 100 & 87.5 \\
\hline 4 & 9 & 90 & 80 & 60 & 100 & 82.5 \\
\hline 5 & 12 & 80 & 100 & 100 & 100 & 95 \\
\hline 6 & 16 & 90 & 100 & 0 & 100 & 72.5 \\
\hline 7 & 20 & 100 & 80 & 10 & 100 & 72.5 \\
\hline 8 & 25 & 40 & 100 & 100 & 100 & 85 \\
\hline 9 & 30 & 70 & 90 & 80 & 100 & 85 \\
\hline 10 & 36 & 70 & 90 & 100 & 100 & 90 \\
\hline
\end{tabular}

\section{ANALYSIS}

Automated discrimination of palmprint images belonging to 4 classes is done using Zernike moments and the best accuracy of $100 \%$ is obtained. To put the above results in perspective with the state of the art, in [6] authors report an accuracy of 96\% using 400 images, in [7] authors report over 99\% accuracy when tested on 1440 images, in [8] authors claim the error rate to be below 3\%, in [10] an acceptance rate of $97 \%$ is reported, in [11] a correct recognition rate of $98 \%$ is reported, in [13] an accuracy of $99 \%$ is reported.

To study the recognition process further, in the second part of the work the original image is segmented to isolate and extract the most relevant part of the image, discarding the other irrelevant part of the image. This is depicted as Region of Interest (ROI) as shown below in Figure 6. Zernike moments are then extracted from the ROI instead of the original image.
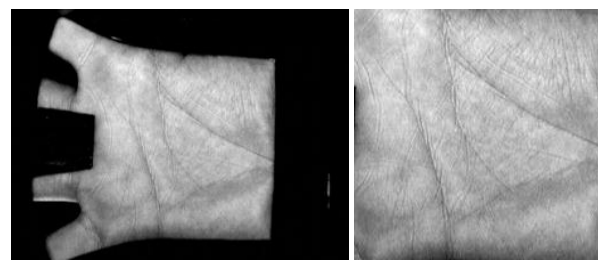

Fig 6: ROI extracted from original image

Recognition accuracies with ROI extracted from original image are tabulated in Table 2 vis-à-vis the corresponding values without the ROI. Although the best results of $100 \%$ are obtained without ROI, accuracies are more consistent with lower fluctuations when ROI is considered. Also overall accuracy when all orders 1 to 10 are considered, are observed to be better $(91.25 \%)$ with ROI than without ROI $(83.75 \%)$. Also the lowest value with ROI is $90 \%$ compared to $72.5 \%$ without ROI. Hence seems to introduce more stability to the experimental results.
Table 2. Comparison with and without ROI

\begin{tabular}{|c|c|c|}
\hline Order & $\begin{array}{c}\text { \% Accuracy } \\
\text { (w/o ROI) }\end{array}$ & $\begin{array}{c}\text { \% Accuracy } \\
\text { (with ROI) }\end{array}$ \\
\hline 1 & 100 & 95 \\
\hline 2 & 67.5 & 95 \\
\hline 3 & 87.5 & 92.5 \\
\hline 4 & 82.5 & 90 \\
\hline 5 & 95 & 90 \\
\hline 6 & 72.5 & 90 \\
\hline 7 & 72.5 & 90 \\
\hline 8 & 85 & 90 \\
\hline 9 & 85 & 90 \\
\hline 10 & 90 & 90 \\
\hline Av & 83.75 & 91.25 \\
\hline
\end{tabular}

Figure 7 shows how accuracy varies with moment order for both 'with ROI' and 'without ROI' cases. I can be concluded that when ROI is used, the results demonstrate more stability and lesser fluctuations with varying Zernike moment orders.

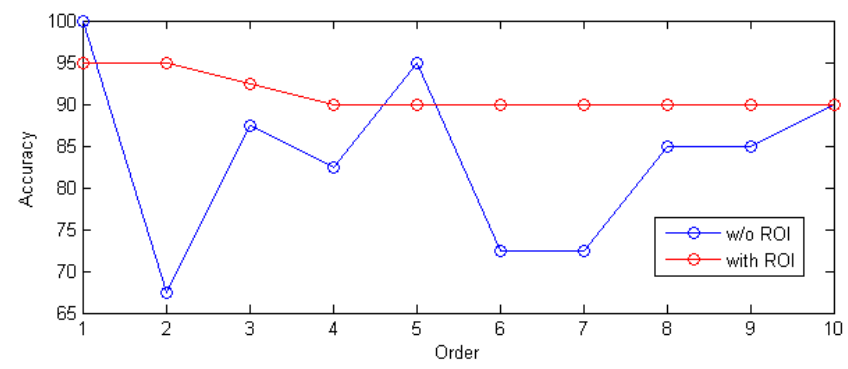

Fig 7: Accuracies with and w/o ROI for different order values

\section{CONCLUSIONS \& FUTURE SCOPES}

This paper proposes an efficient algorithm for palm print recognition using Zernike moments. It seems that using the Zernike moment of order 1 produces better result when compared to using higher order Zernike moments, resulting in better efficiency and lower time complexities. Such methods can prove extremely useful for quick, simple and efficient classification for palm print recognition. Also since the proposed method involves only scalar values instead of multidimensional vectors, it involves reduced requirements of computational resources.

Future work can involve combining Zernike moments with other methods like Moment Invariance and Legendre Moments for improving recognition accuracies. Also statistical classifiers like neural network can be used for more robust classifications. 


\section{REFERENCES}

[1] Mostafa.Abu, Y. S and Psaltis.D. 1985. Image normalization by complex moments. IEEE Trans. On Pattern Analysis and Machine Intelligence, 7(1), pp. 4655.

[2] Dudani, S. A, Breeding.K. J and McGhee.R. B. 1983. Aircraft identification by moment invariants. IEEE Trans. Computers, 26(1), pp. 39-45.

[3] Gayathri, R. and Ramamoorthy.P. 2012. Automatic Palmprint Identification based on High Order Zernike Moment. American Journal of Applied Sciences, pp. 759-765.

[4] Ali.Mian Mahmood, Ghafoor.Mubeen and Taj.Imtiaz A. 2011. Palm Print Recognition Using Oriented Hausdorff Distance, IEEE Proceedings of the 2011 Frontiers of Information Technology. pp. 85-88.

[5] Akinbile.M. O. Rotinwa, Aibinu.A.M. and Salami.M. J. E. 2011. Palmprint Recognition Using Principal Lines Characterization. IEEE First International Conference on Informatics and Computational Intelligence. pp. 278-282.

[6] Yang.Wang-li and Wang.Li-li. 2010. Research of PalmPrint Identification Method Using Zernike Moment and Neural Network. IEEE Sixth International Conference on Natural Computation. pp. 1310-1313.

[7] Fuertes.Juan Jose, Travieso.Carlos M, Ferrer.Miguel A. and Alonso.Jesus B. 2010. Intra-Modal Biometric System Using Hand-Geometry and Palmprint Texture. IEEE International Carnahan Conference on Security Technology. pp. 318-322.

[8] Su.Ching-Liang. 2009. Palm extraction and identification. ELSEVIER Expert Systems with Applications. pp. 1082-1091.

[9] Du.Ning, Qi.Miao, Zhang.Yinan and Kong.Jun. 2009. Palmprint Verification based on Specific-user. IEEE Second International Symposium on Electronic Commerce and Security. pp. 314-317.
[10] Masood.Hassan, Mumtaz.Mustafa, Butt.M Asif Afzal, Mansoor Atif Bin and Khan Shoab A. 2008. Wavelet Based Palmprint Authentication System. IEEE International Symposium on Biometrics and Security Technologies. pp. 1-7.

[11] Michael Goh Kah Ong, Connie Tee and Teoh Andrew Beng Jin. 2008. Touch-less palm print biometrics: Novel design and implementation. ELSEVIER Image and Vision Computing. pp. 1551-1560.

[12] Huang De-Shuang, Jia Wei and Zhang David. 2008. Palmprint verification based on principal lines. ELSEVIER Pattern Recognition. pp. 1316-1328.

[13] Hennings Pablo, Savvides Marios and Kumar B.V.K Vijaya. 2007. Palmprint Recognition with Multiple Correlation Filters Using Edge Detection for ClassSpecific Segmentation. IEEE Workshop on Automatic Identification Advanced Technologies. pp. 214-219.

[14] Pang Ying-Han, Andrew T.B.J, David N.C.L and Hiew Fu San. 2004. Palmprint Verification with Moments. $12^{\text {th }}$ Int. Conf. of Winter School of Computer Graphics (WSCG), pp. 325-332

[15] Sudha Gnanou Florence, Niveditha.M, Srinandhini K., and Narmadha.S. 2011. Hand Based Biometric Recognition Based on Zernike Moments and Log Gabor Filters. International Journal of Research and Reviews in Information Sciences (IJRRIS). pp. 119-125.

[16] Teague, M. R., 1980. Image analysis via the general theory of moments. Journal of Optical Society of America. 70(8), pp. 920-930.

[17] Zernike, F., Physica, vol. 1, p.689, 1934

[18] PolyU palmprint database: (http://www4.comp.polyu.edu.hk/ biometrics/index_db. htm). 